\title{
Is It Possible To Reattach The Retrieved Tooth Fragment From Lower Lip?
}

J osna Vinutha $Y^{1}$

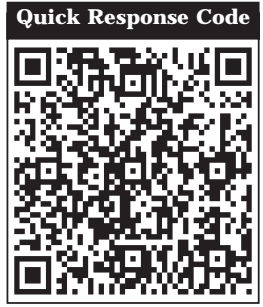

doi: $10.5866 / 2015.7 .10070$

${ }^{1}$ Assistant Professor

Department of Pedodontics \& Preventive Dentistry Army College of Dental Sciences

Secunderabad.

\section{Article Info:}

Received: J anuary 14, 2015

Review Completed: February 12, 2015

Accepted: March 13, 2015

Available Online: April, 2015 (www.nacd.in)

(c) NAD, 2015 - All rights reserved

\section{Email for correspondence:}

dentist4child@gmail.com

\begin{abstract}
:
Trauma to teeth is a common situation in a child patient; every dental professional must be prepared to educate, assess and treat when necessary. It may not only cause physical damage but also affect the patient psychologically. A number of techniques have been developed to restore the fractured tooth. The main purpose of the present case is to present a case of a child who sustained a complicated crown fracture, with the lost portion of tooth embedded in his lower lip. Tooth fragment was removed and successfully reattached to the tooth using acid etch composite resin technique.
\end{abstract}

Key words: Composite resin, Dental trauma, Tooth Fragment Reattachment.

The introduction of composite restorative materials in combination with the use of the acidetch technique to bond composite to enamel made possible the restoration of the fractured incisor with little or no additional tooth preparation. ${ }^{5,6}$ The purpose of this article is to discuss the considerations for dental fragment reattachment technique and to report a case of tooth fragment reattachment after retrieval from the lower lip.

\section{CASE REPORT}

A 12-year-old male patient reported to Department of Pedodontics and Preventive Dentistry, Government Dental College \& Hospital, Hyderabad, following trauma to the maxillary central incisor. Trauma occurred due to fall while cycling two months ago. The parents showed concern about the esthetics of the child.

etch technique. ${ }^{3}$ Subsequently, Stalk
Simonsen have reported similar case. ${ }^{4} \mathrm{~T}$ 
On inspection a swelling on the left side of lip was noticed. A firm nodule measuring approximately $1.5 \mathrm{~cm}$ in diameter in the same region was pal pated. Intra oral examination revealed a horizontally fractured (involving enamel, dentin and pulp) left maxillary permanent central incisor (Figure 1). No mobility of the concerned tooth was recorded and surrounding tissues were healthy. A periapical radiograph showed that the root formation was complete with no extrusion and there were no other injuries. Tooth showed no vitality for pulp tests. Radiograph of the lip confirmed the presence of a tooth fragment in the lower lip (Figure 2). The treatment plan was surgical removal of the tooth fragment from the lip and reattachment of the fragment to the tooth following root canal therapy.

\section{Surgical removal of the tooth fragment from the lip}

Under local anesthesia the patient was submitted to surgical excision of the fragment. The lower lip was incised, tissues were reflected and the tooth fragment was located (Figure 3).

\section{Reattachment of the fragment to the tooth following root canal treatment}

After placement of a rubber dam, pulp was extirpated and following instrumentation the canal was obturated. The access canal was sealed with a glass ionomer plug (VitrebondTM, 3M ESPE, St. Paul, USA). The adaptation of the fragment was checked. Phosphoric acid gel 37.5\% (Scotch BondTM, 3M ESPE, St. Paul, USA) was applied to the enamel of the fragment and the tooth for 20 seconds, limited to $2 \mathrm{~mm}$ beyond the fracture margin. Air-water spray was used to remove the acid and the surface was air-dried. An adhesive bond (Adper Single Bond PIUSTM, 3M ESPE) was applied to the tooth fragment, which was then reattached to its proper position. The fragment was properly positioned on the fractured tooth surface, excess resin was removed and the area was light cured for 60 seconds whilethe fragment was held in place under pressure. The tooth was polished with polishing discs and the rubber dam was removed.

After 6 months follow-up, the repaired tooth showed satisfactory aspect with no periapical changes. During this period, the reattached fragment was intact without any distortion (Figure 4). Six month follow-up showed a predictable outcome of the reattached fragment.

\section{DISCUSSION}

Fracture of teeth may be a most common traumatic incident for a young patient, but it has been found that there is a positive emotional and social response from the patient to the preservation of natural tooth structure. ${ }^{7}$ Theincidence of anterior teeth crown fractures in the permanent dentition is about $26-76 \% .^{8}$

The clinical examination and radiographic evaluation of the patients that missed partially or totally their teeth after traumatic injury is very important, as long as teeth and dental structures may become foreign bodies at risk for ingestion, embedment in surrounding tissue or aspiration. The worst complication is aspiration of foreign bodies that can lead the patient to a variety of chronic airway problems and even death if not precociously diagnosed. ${ }^{9}$ Another important factor is the differential diagnosis, mainly in delayed traumas, because the radiographic image of dental fragments included in the mouth floor can be similar to sialolithiasis of the salivary glands. ${ }^{10}$

In the present case, swelling of the lower lip was observed clinically without pain and the tooth fragment was embedded in the lower lip which was confirmed by the radiograph. There are few published reports of tooth fragments embedded in lip. ${ }^{10}, 11$ The reattachment procedure is a conservative, simple and esthetic treatment option. This technique is less time consuming and provides a more predictable long-term outcome by restoring function and esthetics in a very conservative approach. Pulpal lesion should be diagnosed when the restoration of fractured anterior teeth is considered. The success of reattachment procedure will depend on pulp vitality of the tooth, endodontic treatment of thetooth and amount of tooth structure remaining.

The case reported here describes the surgical removal of tooth fragment and its reattachment with acid-etch technique. The present case also represents an alternative to the use of composite resin or porcelain to restore fractured anterior tooth. The reattachment of a normal tooth fragment can overcome the problem of unmatched shades and wear associated with various restorative materials and techniques. It also helps to satisfy the patient and reduces the financial burden to the parent. 


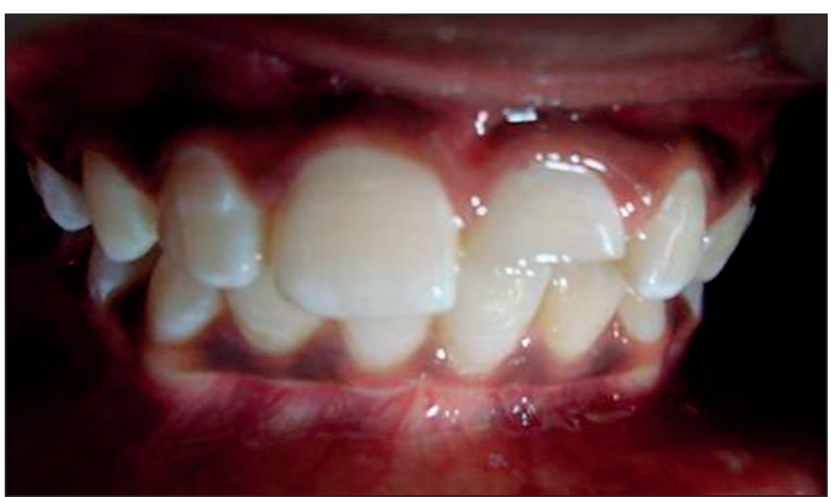

Figure 1: Coronal fracture of maxillary central incisor

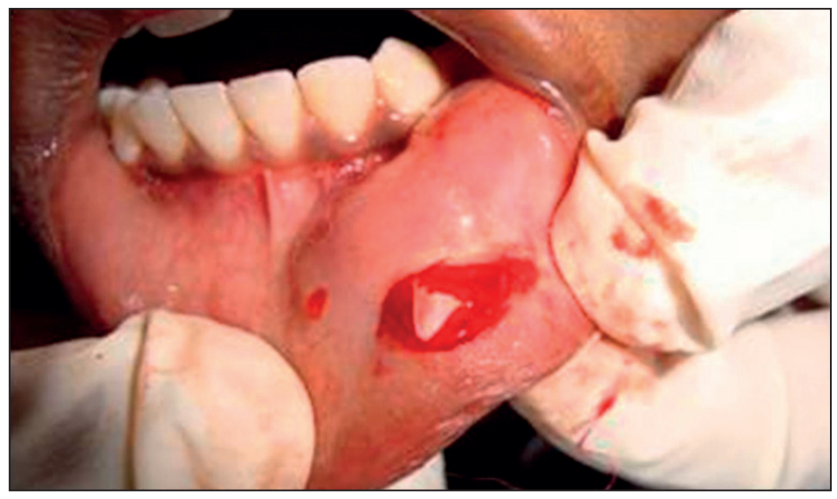

Figure 3: The removed tooth fragment. The tooth fragment was carefully removed and checked for the fit with the tooth; and immediately maintained in normal saline during the whole period prior to restoration.

\section{CONCLUSION}

Tooth fragment reattachment procedure offers ultraconservative, safe, fast, cost effective and esthetically pleasing results. Detailed case history and proper clinical examination should be taken to locate the fractured fragment. Radiographs should be taken for confirmation. The reattachment of the tooth fragment as a restorative procedure becomes possible only when the missing fragment is available and the tooth is restorable. Various adhesive techniques and restorative materials can be used to improve this procedure.

\section{REFERENCES}

1. Rober tson A, Rober tson S, Noren J G. A restorative evaluation of traumatized permanent teeth. Int J Paed Dent 1997; 7(4):217-226.

2. Murchinson DF, Burke FJ T, Worthington RB. Incisal edge reattachment; indications for use and clinical technique. Br Dent J 1999; 186(12):614-619.

3. Tennery NT. The fractured tooth reunited using the acid etch bonding technique. Tex Dent J 1978; 96(8):16-17.

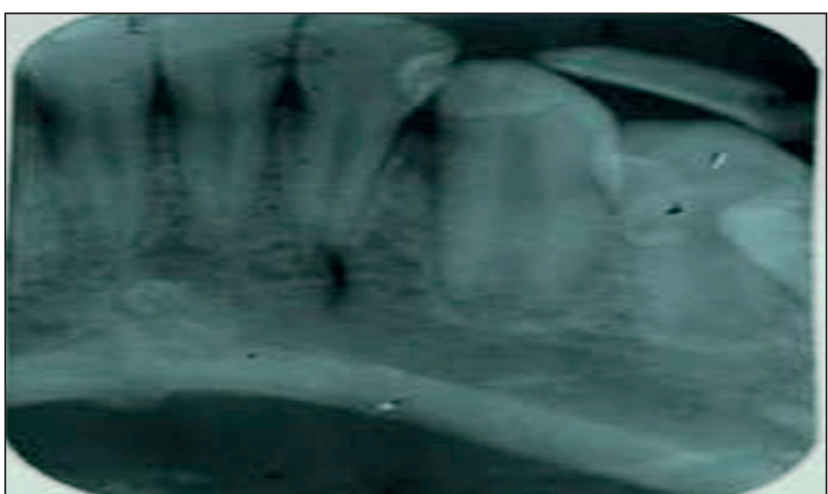

Figure 2: I mage showing the tooth fragment in the lower lip.

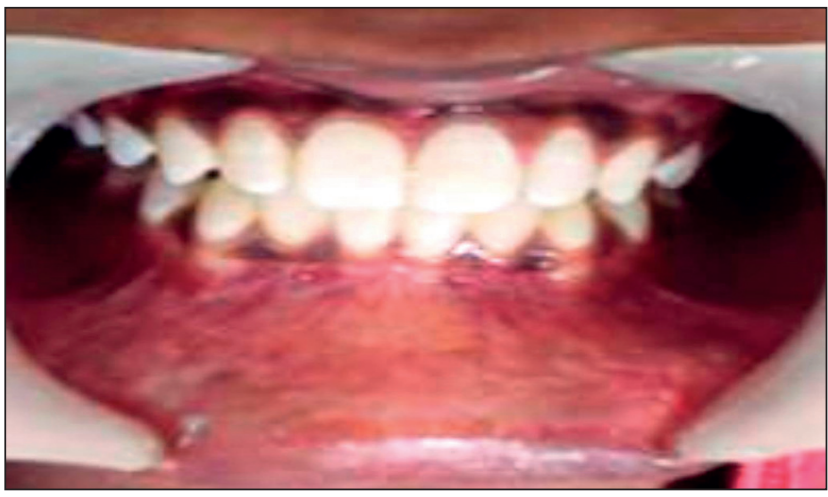

Figure 4: Tooth fragment reattached, showing good aesthetic results.

4. Starkey PE. Reattachment of a fractured fragment to a tooth. J Indiana Dent Assoc1979; 58(5):37-38.

5. Hegde RJ . Tooth fragment reattachment - an esthetic alternative: Report of a case. J Indian Soc Pedod Prev Dent 2003; 21(3):117-119.

6. Burke FJ T. Reattachment of a fractured central incisor tooth fragment. Br Dent J 1991; 170(6):223-225.

7. Hamilton FA, Hill FJ, Holloway PJ . An investigation of dento-alveolar trauma and its treatment in an adolescent population. Part-1: The prevalence and incidence of injuries and the extent and adequacy of treatment received. $\mathrm{Br}$ Dent J 1997; 182:91-95.

8. Simonsen RJ . Restoration of a fractured central incisor using original teeth. J Am Dent Assoc 1982; 105(4):646648.

9. Kimberly DR. Unrecognized aspiration of a mandibular incisor. J Oral Maxillofac Surg 2001; 59(3):350-352.

10. da Silva AC, de Moraes M, Bastos EG, Moreira RWF, Passeri LA. Tooth fragment embedded in the lower lip after dental trauma: case reports. Dent Traumatol 2005; 21(2):115-120.

11. Naudi AB, Fung DE. Tooth Fragment reattachment after retrieval from the lower lip - a case report. Dent Traumatol 2007; 23(3):177-180. 